\title{
Ultrasonict
}

Ultrasonics 36 (1998) 803-811

\section{Locating the crack tip of a surface-breaking crack Part I. Line crack}

\author{
M.K. Kuo *, T.R. Lin, P.L. Liu, T.T. Wu \\ Institute of Applied Mechanics, National Taiwan University, Taipei, Taiwan 106
}

Received 10 December 1997; received in revised form 20 January 1998

\begin{abstract}
Four distinct algorithms to locate the crack tip of a surface-breaking crack using only the arrival time information of the first diffracted waves are described and compared. To illustrate these algorithms, a line crack in a half-plane is considered. The first two algorithms are based mainly on elementary geometric arguments, where the crack tip is formulated as the intersecting point of two ellipses (algorithm 1) and/or three circles (algorithm 2). The other two algorithms are formulated as optimization problems, where cost functions based upon the arrival time data of diffracted waves are constructed. The unknown crack tip coordinates are then determined by minimizing the cost functions through the Lagrange multiplier method (algorithm 3) or the simplex method (algorithm 4). In the numerical experiments, the exact arrival times are superimposed by Gaussian error with different levels to simulate the real extracted arrival times from experimental signals. The numerical optimization method (algorithm 4 ) is found to have the best performance with respect to noise, as well as for accuracy. Moreover, the recovery of the crack length is much more robust than the orientation and depth. (C) 1998 Elsevier Science B.V. All rights reserved.
\end{abstract}

Keywords: Crack length measurement; Time-of-flight diffraction; Non-destructive evaluation; Crack tip localization

PACS: 43.35.Z; 81.70.D; 43.66.Q

\section{Introduction}

The detection of cracks and the determination of their location, size, shape and orientation are of great interest in the field of quantitative non-destructive evaluation of materials, since they serve as important fracture mechanics parameters in the failure prediction of structural components. Possible roles that ultrasonic non-destructive evaluation could play in the prevention of failure have been described by Achenbach [1]. Ultrasonic bulk and surface waves have been routinely used in the detection of cracks as well as in the depth measurement of surface-breaking cracks in metallic materials. For the measurement of the depth and/or length of cracks, most methods have been based on the frequency content of the reflected and/or scattered waves [2,3], or time-offlight of bulk and/or surface waves developed mainly by Silk [4-10]. A review on ultrasonic crack depth measurement has been reported by Doyle and Scala [11].

* Corresponding author. Tel.: (886) 22363 0979, ext. 318

fax.: (886) 22366 1446; e-mail: mkkuo@ccms,ntu.edu.tw
However, in some materials such as concrete, the use of impact sources is preferred over single-frequency ultrasonic wavestrains in order to have enough depth penetration into the specimen. Sansalone and co-workers $[12,13]$ developed the impact-echo method to detect the depth of surface-breaking cracks in beams and columns of concrete. This method was based on the frequencies of the recorded surface displacement waveforms. Lin and Su [14] used the same method to detect the depth of a vertical crack in concrete structures. This problem was also addressed by $\mathrm{Wu}$ et al. [15] using phase information. Recently, Liu et al. [16] adopted the idea used in geophysical migration to image a surfacebreaking crack in two-dimensional concrete, again by using impact force. The basic principle was to align the source and the receivers geometrically on either side of the surface-breaking cracks, which is similar to the timeof-flight diffraction (TOFD) [4-10] but in contrast to the impact-echo method [12-14]. The tip of the crack was successfully resolved, and the actual depth of the crack could then be calculated accordingly.

In this paper, methods based on the arrival times of 
diffracted impact-induced transient elastic waves to detect the tip of an inclined surface-breaking crack are proposed. Knowing the location of the source and receiver, as well as the wave speed of the specimen, four different algorithms recovering the crack tip and hence the depth, length and orientation of surface-breaking cracks are presented and compared. To illustrate the algorithms, only the cases of line cracks in a half plane are presented here. The primary interest here are cracks with lengths of the order of $10 \mathrm{~cm}$ in plane concrete. Hence the use of impact force is preferred, which will then limit the possibility of scan processes used in TOFD. The main difference between these methods and TOFD are the source-receiver configuration and the processing of the arrival time data. In TOFD, a singlesource and single-receiver configuration is used together with a linear scan on the surface of the specimen. Hence a plot of B-scan result is needed with a curve fitting or synthesis aperture focusing technique to locate the crack tip. However, two sets of single source, each with multiple receivers but no scan, are used in this paper. The arrival time data are then directly extracted from the receiving signals to process further. The results show that the proposed methods can successfully recover the crack tip, and hence the length, depth, and orientation of a surface-breaking crack. Since the methods rely only on the arrival times of first diffracted waves, they can be applied directly to any real structural components with a flat surface. Moreover, the methods are reliable, quick and accurate. These are the main elements to apply the artificial intelligence in crack-clepth measurements [17]. The extension of these methods to planar cracks in hall space will be reported elsewhere.

\section{Statement of problem}

Consider a homogeneous, isotropic, linearly elastic half-plane containing a surface-breaking line crack of arbitrary orientation. The crack tip coordinates are denoted by $\left(x_{\mathrm{c}}, y_{\mathrm{c}}\right)$. Cartesian coordinates is defined in such a way that the half-plane is in the region of $y>0$, and the surface of the half-pane coincides with the axis of $x$. The coordinate system, the crack geometry and the half-plane are shown in Fig. 1.

The elastic half-plane is initially at rest, for $t<0$. For time $t \geq 0$, an impact normal force $F(t)$, e.g. dropping a small steel ball onto a specimen, acts on the free surface of the half-plane at the point $(x, y)=\left(x_{\mathrm{s}}, 0\right)$ on one side of the crack mouth. In the mean time, there are $n$ receivers, which are located at $\left(x_{i}, 0\right), i=1, \ldots, n$, on the other side of the crack mouth. The arrival time, $t_{i}$, of the first diffracted wave from the loading point to the crack tip and then to the $i$ th receiver is

$t_{\mathrm{i}}=S_{\mathrm{p}}\left\{\left[\left(x_{\mathrm{s}}-x_{\mathrm{c}}\right)^{2}+y_{\mathrm{c}}^{2}\right]^{1 / 2}+\left[\left(x_{\mathrm{c}}-x_{i}\right)^{2}+y_{\mathrm{c}}^{2}\right]^{1 / 2}\right\}$

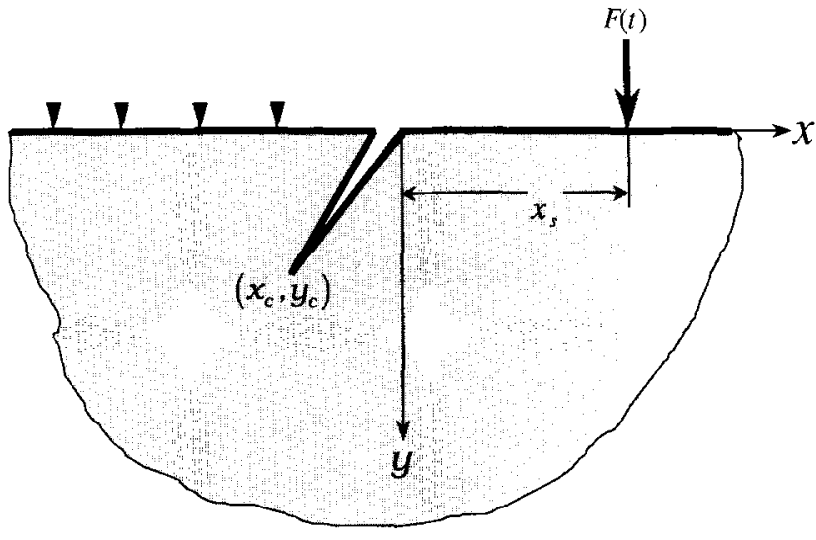

Fig. 1. Geometry of the cracked half-plane.

where $s_{\mathrm{p}}=1 / c_{\mathrm{p}}$ is the slowness of longitudinal wave of the half-plane.

In TOFD, a single source and single receiver configuration is used together with a linear scan over the surface of the body. In this paper, a crack length of the order of $10 \mathrm{~cm}$ in plain concrete is of primary interest. The configuration of multiple receivers with each single impact force is considered, but no scan. In fact, it is more reasonable to use two single-source/multiplereceiver configuration separately for each complete experiment, one for the source to the right of the crack mouth and another for the source to the left of the crack mouth. This will address the problem of whether the source and the crack tip are on the same side or different sides of the crack mouth. This source-receiver configuration is also different from the impact-ccho method [12-14], where the source and receiver are on the same side of the crack mouth.

Suppose we have extracted the arrival times, $t_{i}$, from experimental responses, and that we have knowledge of the wave speed of the specimen by other means, e.g. [18]. We then try to identify the crack tip coordinates from $t_{i}$.

\section{Methods of solution}

The extracted arrival-time data from experimental responses will probably not be exact. We propose two catalogues of methods to locate the crack tip under this situation. Sections 3.1 and 3.2 in the first catalogue are in two steps, based on elementary geometric arguments. In the first step, possible candidates $\left(X_{\mathrm{c}}, Y_{\mathrm{c}}\right)$ of the crack tip are constructed by geometric arguments from all possible pairs of two receivers with respect to the same source. A conclusive crack tip $\left(x_{c}, y_{c}\right)$ is then reached in the second step by a simple algebraic average of all $\left(X_{\mathrm{c}}, Y_{\mathrm{c}}\right)$. The second catalogue of methods, Sections 3.3 and 3.4, formulate the problem into optimization prob- 
lems where $\left(x_{\mathrm{c}}, y_{\mathrm{c}}\right)$ is adjusted to best fit the arrival data $t_{i}$.

\subsection{Intersection of half-ellipses}

From an elementary geometric viewpoint, i.e. where the locus of all possible points of $\left(X_{\mathrm{c}}, Y_{\mathrm{c}}\right)$ with $Y_{\mathrm{c}}>0$, where the sum of distances from $\left(x_{\mathrm{s}}, 0\right)$ to $\left(X_{\mathrm{c}}, Y_{\mathrm{c}}\right)$ and from $\left(X_{\mathrm{c}}, Y_{\mathrm{c}}\right)$ to $\left(x_{i}, 0\right)$ equals $c_{\mathrm{p}} t_{i}$, will define a halfellipse with focal points at $\left(x_{i}, 0\right)$ and $\left(x_{s}, 0\right)$ as

$$
\frac{\left(2 X_{\mathrm{c}}-x_{\mathrm{s}}-x_{\mathrm{i}}\right)^{2}}{\left(c_{\mathrm{p}} t_{i}\right)^{2}}+\frac{\left(2 Y_{\mathrm{c}}\right)^{2}}{\left(c_{\mathrm{p}} t_{i}\right)^{2}-\left(x_{\mathrm{s}}-x_{i}\right)^{2}}=1, \quad i=1,2, \ldots, n,
$$

where $\left(X_{\mathrm{c}}, Y_{\mathrm{c}}\right)$ are the coordinates of possible crack tips. Hence, giving the arrival time data of a receiver, namely a pair of $\left(x_{i}, t_{i}\right)$, defines a half-ellipse as in Eq. (2). Consequently, when any two pairs of $\left(x_{i}, t_{i}\right)$ with the same source location are given, the intersecting points of the two corresponding half-ellipses would suggest the position of the actual crack tip, assuming there were no errors in the $t_{i}$. The intersection of two half-ellipses are illustrated in Fig. 2.

Eliminating $Y_{\mathrm{c}}$ from two equations of Eq. (2), say $i=$ 1 and $i=2$ without loss of generality, leads to a quadratic equation of $X_{\mathrm{c}}$. From elementary algebra, the roots of a quadratic equation could be one of the following three cases: two real roots, one repeated real root, or two complex roots, e.g.

$X_{\mathrm{c} \pm}=\frac{-B_{\mathrm{e}} \pm\left(B_{\mathrm{e}}^{2}-A_{\mathrm{e}} D_{\mathrm{e}}\right)^{1 / 2}}{2 A_{\mathrm{e}}}$

where

$A_{\mathrm{e}}=a_{\mathrm{e} 2}^{2} b_{\mathrm{e} 1}^{2}-a_{\mathrm{e} 1}^{2} b_{\mathrm{e} 2}^{2}$,

$B_{\mathrm{e}}=-d_{\mathrm{e} 1} a_{\mathrm{e} 2}^{2} b_{\mathrm{e} 1}^{2}+d_{\mathrm{e} 2} a_{1}^{2} b_{\mathrm{e} 2}^{2}$,

$D_{\mathrm{e}}=a_{\mathrm{e} 2}^{2} b_{\mathrm{e} 1}^{2}\left(d_{\mathrm{e} 1}^{2}-a_{\mathrm{e} 1}^{2}\right)-a_{\mathrm{e} 1}^{2} b_{\mathrm{e} 2}^{2}\left(d_{\mathrm{e} 2}^{2}-a_{\mathrm{e} 2}^{2}\right)$,

and $a_{\mathrm{e} i}=c_{\mathrm{p}} t_{i}, b_{\mathrm{e} i}^{2}=\left(c_{\mathrm{p}} t_{i}\right)^{2}-\left(x_{\mathrm{s}}-x_{i}\right)^{2}, d_{\mathrm{e} i}=x_{\mathrm{s}}+x_{i}$. At first glance, we might think that this merely suggests that

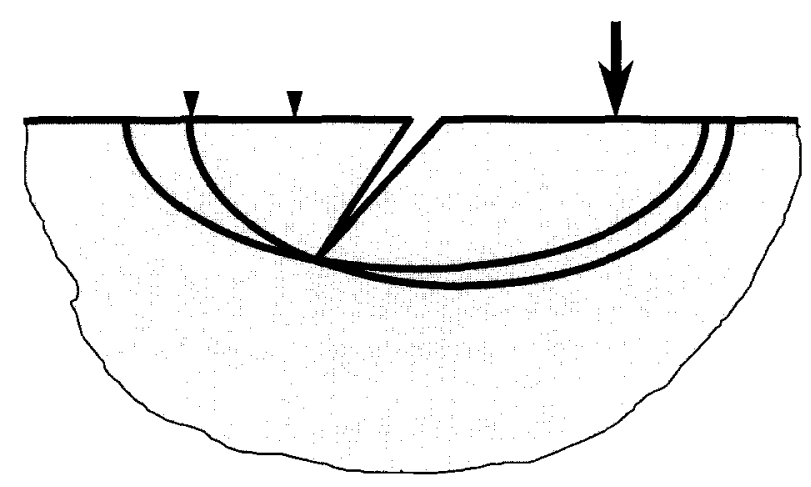

Fig. 2. Illustrating of intersection of two half-ellipses. the numbers of intersecting points of two half-ellipses could be two, one or even none, respectively. On the other hand, the $X_{\mathrm{c} \pm}$ in Eq. (3) has to satisfy a constraint condition

$$
\begin{aligned}
\max \left(d_{\mathrm{e} 1}\right. & \left.-a_{\mathrm{e} 1}, d_{\mathrm{e} 2}-a_{\mathrm{e} 2}\right) \\
& <2 X_{\mathrm{c}}<\min \left(d_{\mathrm{e} 1}+a_{\mathrm{e} 1}, d_{\mathrm{e} 2}+a_{\mathrm{e} 2}\right) .
\end{aligned}
$$

Geometrically, this constraint states that the $x$-coordinate of the intersection point should be bounded by $\left(d_{\mathrm{e} i}-a_{\mathrm{e} i}\right) / 2$ and $\left(d_{\mathrm{e} i}+a_{\mathrm{e} i}\right) / 2, i=1,2$, which arc the smallest and largest $x$-coordinates, respectively, of two original half cllipses. In fact, in almost all of our numerical simulation, the quadratic equations of $X_{\mathrm{c}}$ always have two (if any) real roots, but only one of them satisfies the constraint condition, Eq. (7). Once $X_{\mathrm{c}}$ is known, the vertical coordinate of the crack tip is easily found from Eq. (2):

$Y_{\mathrm{c}}=\frac{b_{\mathrm{e} 1}}{2 a_{\mathrm{c} 1}}\left[a_{\mathrm{e} 1}^{2}-\left(2 X_{\mathrm{c}}-d_{\mathrm{e} 1}\right)^{2}\right]^{1 / 2}$.

The constraint condition on $X_{c}$, Eq. (7), guarantees the positivity of the term inside the bracket on the RHS of Eq. (8). Notice that the candidates of the crack tip determined from each pair of receivers are not likely to be identical, unless all the arrival time data are exact. A further algebraic average over all candidates is employed to yield a conclusive result of $\left(x_{\mathrm{c}}, y_{\mathrm{c}}\right)$.

If the crack is known to be vertical beforehand, i.e. $X_{\mathrm{c}}=x_{\mathrm{c}}=0$, one arrival time data alone or equivalently one equation of Eq. (2) is enough to find the vertical coordinate of the crack tip. The resulting $Y_{c}$ is exactly the same as by allowing $X_{\mathrm{c}}=0$ in Eq. (8). Again, the conclusive result of $y_{\mathrm{c}}$ is achieved by a simple algebraic average over all possible $Y_{\mathrm{c}} \mathrm{s}$.

\subsection{Intersection of half-circles}

If the travel time $t_{0}$ is given, which corresponds to the time needed for a signal to travel from the loading point to the crack tip, the locus of all possible crack tips $\left(X_{\mathrm{c}}, Y_{\mathrm{c}}\right)$, with $Y_{\mathrm{c}}>0$, will form a half-circle centered at the loading point with radius $c_{\mathrm{p}} t_{0}$ as

$\left(X_{\mathrm{c}}-x_{\mathrm{s}}\right)^{2}+Y_{\mathrm{c}}^{2}=c_{\mathrm{p}}^{2} t_{0}^{2}$.

Similarly, the locus of all possible crack tips will also form a half-circle but centered at each receiver with radius $c_{\mathrm{p}}\left(t_{i}-t_{0}\right)$ as

$\left(X_{\mathrm{c}}-x_{i}\right)^{2}+Y_{\mathrm{c}}^{2}=c_{\mathrm{p}}^{2}\left(t_{i}-t_{0}\right)^{2}, \quad i=1,2, \ldots, n$.

Any two half-circles defined by Eq. (10) and one halfcircle defined by Eq. (9) will intersect at the same point, namely the crack tip point, if $t_{\mathrm{O}}$ is correct. Hence Eq. (9) and any two Eq. (10) will serve a system of equations for $X_{c}, Y_{c}$ and $t_{0}$. The intersection of two half-ellipses are shown in Fig. 3. It is worthwhile to note that a 


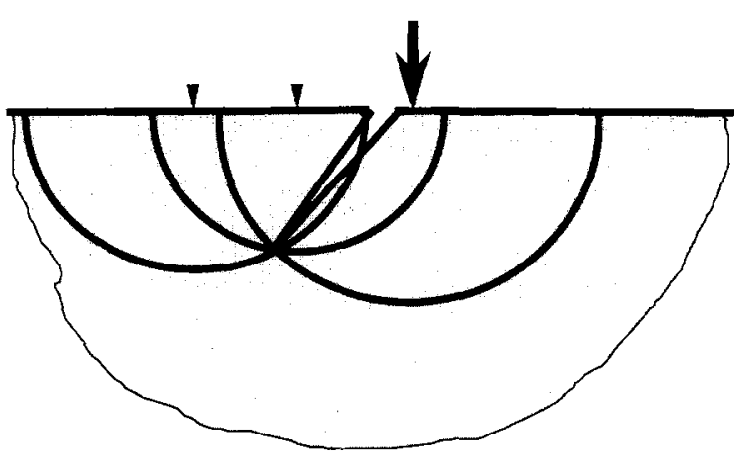

Fig. 3. Illustrating of intersection of three half-circles.

similar idea was reported by [19] using pulse-echo configuration where the travel time from the crack tip to the receiver was known. Hence any two Eq. (10), with $t_{i}-t_{0}$ given from the pulse-echo result, can be used to locate the crack tip.

In our current configuration, $t_{i}-t_{0}$ is not known a priori. Eliminating $Y_{\mathrm{c}}$ from Eq. (9) and from any one of Eq. (10), leads to a linear equation of $X_{\mathrm{c}}$ and $t_{0}$ as

$2\left(x_{\mathrm{s}}-x_{i}\right) X_{\mathrm{c}}+2 t_{i} t_{0} c_{\mathrm{p}}^{2}=c_{\mathrm{p}}^{2} t_{i}^{2}-x_{i}^{2}+x_{\mathrm{s}}^{2}$.

Hence from any two receivers, e.g. $i=1$ and $i=2$ without loss of generality, two of Eq. (11) will give rise to a system of two linear equations for two unknowns $X_{\mathrm{c}}$ and $t_{0}$. The result is straightforward:

$X_{\mathrm{c}}=\left(b_{\mathrm{c} 2} d_{\mathrm{c} 1}-b_{\mathrm{c} 1} d_{\mathrm{c} 2}\right) / \Delta_{\mathrm{c}}$,

$t_{0}=-\left(a_{\mathrm{c} 2} d_{\mathrm{c} 1}-a_{\mathrm{c} 1} d_{\mathrm{c} 2}\right) / \Delta_{\mathrm{c}}$,

where $\Delta_{\mathrm{c}}=a_{\mathrm{c} 1} b_{\mathrm{c} 2}-a_{\mathrm{c} 2} b_{\mathrm{c} 1}, a_{\mathrm{c} i}=2\left(x_{\mathrm{s}}-x_{i}\right), b_{\mathrm{c} i}=2 t_{i} c_{\mathrm{p}}^{2}$ and $d_{\mathrm{c} i}=c_{\mathrm{p}}^{2} t_{i}^{2}-x_{i}^{2}+x_{\mathrm{s}}^{2}$. The expression of $X_{\mathrm{c}}$ in Eq. (12) is definitely much more compact than the one in Eq. (3). Notice that the roots $X_{\mathrm{c}}$ and $t_{0}$ are feasible only if

$$
\begin{aligned}
\max \left(x_{i}-c_{\mathrm{p}} t_{i}+c_{\mathrm{p}} t_{0}, x_{\mathrm{s}}-c_{\mathrm{p}} t_{0}\right)<X_{\mathrm{c}} \\
<\min \left(x_{i}+c_{\mathrm{p}} t_{i}-c_{\mathrm{p}} t_{0}, x_{\mathrm{s}}+c_{\mathrm{p}} t_{0}\right) .
\end{aligned}
$$

Geometrically, this constraint states that the $x$-coordinate of the intersection point, $X_{\mathrm{c}}$, should be bounded by smallest and largest $x$-coordinates of the original three circles. Once $X_{\mathrm{c}}$ and $t_{0}$ are known, the corresponding possible vertical coordinate of the crack tip is

$Y_{\mathrm{c}}=\left[c_{\mathrm{p}}^{2} t_{0}^{2}-\left(x_{\mathrm{s}}-X_{\mathrm{c}}\right)^{2}\right]^{1 / 2}$.

The constraint condition Eq. (14) guarantees the positivity of the term inside the bracket in the RHS of Eq. (15). Again, a simple algebraic average is used to yield conclusive coordinates over all candidates determined from all pairs of receivers.

If the crack is known to be vertical, i.e. $X_{\mathrm{c}}=x_{\mathrm{c}}=0$, Eq. (11) alone is used to find $t_{0}=d_{\mathrm{ci}} / b_{\mathrm{c} r}$. The corresponding possible vertical coordinate of the crack tip is again followed from Eq. (15) with $X_{\mathrm{c}}=0$.

\subsection{Analytical optimization}

From the optimization viewpoint, the methods in Sections 3.1 and 3.2 are in two steps. In the first step, possible candidates $\left(X_{\mathrm{c}}, Y_{\mathrm{c}}\right)$ of the crack tip are determined from all pairs of two receivers. A conclusive crack tip $\left(x_{\mathrm{c}}, y_{\mathrm{c}}\right)$ is then reached in the second step by finding the least-squared error point in the coordinates, i.e. having the least values in the error functions (or cost functions) of $F_{1 x}$ and $F_{1 y}$,

$F_{1 x}=\sum_{X_{\mathrm{c}}}\left(X_{\mathrm{c}}-x_{\mathrm{c}}\right)^{2}$

$F_{1 y}=\sum_{Y_{\mathrm{c}}}\left(Y_{\mathrm{c}}-y_{\mathrm{c}}\right)^{2}$.

It is possible to formulate the problem as a more direct optimization problem by adjusting the coordinates of the crack tip $\left(x_{\mathrm{c}}, y_{\mathrm{c}}\right)$ to minimize the error between estimated and extracted arrival times. One possibility is to construct the error function as

$$
\begin{aligned}
F_{2}\left(x_{\mathrm{c}}, y_{\mathrm{c}}\right)= & \sum_{\mathrm{i}=1}^{n}\left\{\left[\left(x_{\mathrm{s}}-x_{\mathrm{c}}\right)^{2}+y_{\mathrm{c}}^{2}\right]^{1 / 2}\right. \\
& \left.+\left[\left(x_{\mathrm{c}}-x_{i}\right)^{2}+y_{\mathrm{c}}^{2}\right]^{1 / 2}-c_{\mathrm{p}} t_{i}\right\}^{2} .
\end{aligned}
$$

If all $t_{i}$ were exact, the true $\left(x_{\mathrm{c}}, y_{\mathrm{c}}\right)$ will certainly minimize the value of function $F_{2}$, which will be zero. The minimization of Eq. (18) certainly deserves a numerical approach.

With the aid of the geometric analysis in Section 3.2, it is possible to construct an error function, which is a function of $x_{c}, y_{c}$ and $t_{0}$, as

$F_{3}\left(x_{\mathrm{c}}, y_{\mathrm{c}}, t_{0}\right)=\sum_{i=1}^{n}\left[\left(x_{i}-x_{\mathrm{c}}\right)^{2}+y_{\mathrm{c}}^{2}-c_{\mathrm{p}}^{2}\left(t_{i}-t_{0}\right)^{2}\right]^{2}$

subjected to a constraint condition

$\Phi\left(x_{\mathrm{c}}, y_{\mathrm{c}}, t_{0}\right) \equiv\left(x_{\mathrm{s}}-x_{\mathrm{c}}\right)^{2}+y_{\mathrm{c}}^{2}-c_{\mathrm{p}}^{2} t_{0}^{2}=0$.

Again, if all $t_{i}$ were exact, the true $x_{\mathrm{c}}, y_{\mathrm{c}}$ and $t_{0}$ will certainly minimize the value of function $F_{3}$, which will be zero, and satisfy the constraint condition Eq. (20). Since both $F_{3}$ and $\Phi$ have continuous first partial derivatives, the constrained optimization problem defined by Eqs. (19) and (20) can be solved by the Lagrange multiplier method. We can construct a new functional as

$F_{4}\left(x_{\mathrm{c}}, y_{\mathrm{c}}, t_{0} ; \lambda, \Phi\right)=F_{3}\left(x_{\mathrm{c}}, y_{\mathrm{c}}, t_{0}\right)+\lambda \Phi\left(x_{\mathrm{c}}, y_{\mathrm{c}}, t_{0}\right)$

where $\lambda$ is a constant. We can find $x_{\mathrm{c}}, y_{\mathrm{c}}, t_{0}$ and $\lambda$ from the conditions when the partial derivatives of $F_{4}$ with respect to $x_{\mathrm{c}}, y_{\mathrm{c}}$ and $t_{0}$, respectively, equal zero, i.e.

$2 \sum_{i=1}^{n}\left[\left(x_{i}-x_{\mathrm{c}}\right)^{2}+y_{\mathrm{c}}^{2}-c_{\mathrm{p}}^{2}\left(t_{i}-t_{0}\right)^{2}\right]\left(x_{i}-x_{\mathrm{c}}\right)$
$\quad+i\left(x_{\mathrm{s}}-x_{\mathrm{c}}\right)=0$, 
$2 \sum_{i=1}^{n}\left[\left(x_{i}-x_{\mathrm{c}}\right)^{2}+y_{\mathrm{c}}^{2}-c_{\mathrm{p}}^{2}\left(t_{i}-t_{0}\right)^{2}\right] y_{\mathrm{c}}+\lambda y_{\mathrm{c}}=0$,

$2 \sum_{i=1}^{n}\left[\left(x_{i}-x_{\mathrm{v}}\right)^{2}+y_{\mathrm{v}}^{2}-c_{\mathrm{p}}^{2}\left(t_{i}-t_{0}\right)^{2}\right]\left(t_{i}-t_{0}\right)-\lambda t_{0}=0$,

as well as the original constraint condition Eq. (20). Notice that Eqs. (22)-(24) amount to minimizing $F_{4}$ subject to no constraints.

The Lagrange multiplier $\lambda$ can now be easily expressed in terms of $x_{\mathrm{c}}, y_{\mathrm{c}}$ and $t_{0}$ by using Eq. (23) provided $y_{\mathrm{c}} \neq 0$. Substituting $\lambda$ into Eqs. (22) and (24) and making use of the constraint condition Eq. (20), yields two simultaneous linear equations for $x_{\mathrm{c}}$ and $t_{0}$. We then have

$x_{\mathrm{c}}=\left(D_{\mathrm{a} 1} B_{\mathrm{a} 2}-D_{\mathrm{a} 2} B_{\mathrm{a} 1}\right) / A_{\mathrm{a}}$,

$t_{0}=\left(A_{\mathrm{a} 1} D_{\mathrm{a} 2}-A_{\mathrm{a} 2} D_{\mathrm{a} 1}\right) / A_{\mathrm{a}}$,

where $A_{\mathrm{a}}=A_{\mathrm{a} 1} B_{\mathrm{a} 2}-A_{\mathrm{a} 2} B_{\mathrm{a} 1}$. The coefficients $A_{\mathrm{a} 1}$, $A_{\mathrm{a} 2}, \ldots$ are defined as

$$
\left\{\begin{array}{l}
A_{\mathrm{a} 1} \\
B_{\mathrm{a} 1} \\
D_{\mathrm{a} 1}
\end{array}\right\}=\sum_{i=1}^{n}\left\{\begin{array}{l}
a_{\mathrm{c} i} \\
b_{\mathrm{c} i} \\
d_{\mathrm{c} i}
\end{array}\right\}\left(x_{\mathrm{s}}-x_{i}\right), \quad\left\{\begin{array}{l}
A_{\mathrm{a} 2} \\
B_{\mathrm{a} 2} \\
D_{\mathrm{a} 2}
\end{array}\right\}=\sum_{i=1}^{n}\left\{\begin{array}{l}
a_{\mathrm{c} i} \\
b_{\mathrm{c} i} \\
d_{\mathrm{c} i}
\end{array}\right\} t_{i}
$$

where $a_{\mathrm{c} i}, b_{\mathrm{ci}}$ and $d_{\mathrm{ci}}$ are the same as defined in Section 3.2. Again, the feasible roots of $x_{\mathrm{c}}$ and $t_{0}$ are guaranteed only if Eq. (14) is satisfied. Once $x_{\mathrm{c}}$ and $t_{0}$ are known, Eq. (15) will again give the vertical coordinate of the crack tip.

Notice that the results of Eqs. (25) and (26) are valid for cases of any single-source/multiple-receiver configuration. In the current numerical experiments, there are always two source points, one per each sct of arrival time data. Of course, the error function Eq. (19) can be extended into such configurations subjected to two corresponding constraint conditions. However, the complexity of the equations may elude the possibility of simple analytic results for $x_{\mathrm{c}}, y_{\mathrm{c}}$ and $t_{0}$ as in Eqs. (25) and (26). We again employ a two-step process, i.e., finding $\left(x_{c k}, y_{c k}, t_{0 k}\right), k=1,2$, according to Eqs. (25) and (26) for each set of arrival times. Then a simple algebraic average is employed to yield a conclusive crack tip $\left(x_{\mathrm{c}}, y_{\mathrm{c}}\right)$.

If the crack is known to be vertical, i.e. $x_{\mathrm{c}}=0$, the Lagrange multiplier $\lambda$ is again expressed in terms of $y_{c}$ and $t_{0}$ using Eq. (23). Substituting $\lambda$ into Eq. (24) alone and making use of the constraint condition Eq. (20), yields a single linear equation for $t_{0}$. Hence we have $t_{0}=D_{\mathrm{a} 2} / B_{\mathrm{a} 2}$. The vertical coordinate of the crack tip is again followed from Eq. (15) with $x_{\mathrm{c}}=0$.

\subsection{Numerical optimization}

If the error function takes a form other than Eq. (19), simple analytic expressions of $x_{\mathrm{c}}$ and $y_{\mathrm{c}}$ may not be available. The recovery of crack tip coordinates can still be achieved by employing a stable numerical optimization algorithm such that the difference between the estimated and the extracted arrival times reaches a best fit.

The error function $F_{2}$ described in Eq. (18) of Section 3.3 is normalized further and modified to take into account the fact that there are two sources per each complete experiment

$$
\begin{aligned}
& F_{5}\left(x_{\mathrm{c}}, y_{\mathrm{c}}\right) \\
& =\sum_{i=1}^{n 1}\left\{\frac{\left[\left(x_{\mathrm{s} 1}-x_{\mathrm{c}}\right)^{2}+y_{\mathrm{c}}^{2}\right]^{1 / 2}+\left[\left(x_{\mathrm{c}}-x_{i 1}\right)^{2}+y_{\mathrm{c}}^{2}\right]^{1 / 2}}{c_{\mathrm{p}} t_{i}}-1\right\}^{2} \\
& +\sum_{j=1}^{n 2}\left\{\frac{\left[\left(x_{\mathrm{s} 2}-x_{\mathrm{c}}\right)^{2}+y_{\mathrm{c} 2}\right]^{1 / 2}+\left[\left(x_{\mathrm{c}}-x_{j 2}\right)^{2}+y_{\mathrm{c} 2}\right]^{1 / 2}}{c_{\mathrm{p}} t_{j}}-1\right\}^{2}
\end{aligned}
$$

where $\left(x_{\mathrm{s} 1}, 0\right)$ and $\left(x_{\mathrm{s} 2}, 0\right)$ are the coordinates of the sources, and $\left(x_{i 1}, 0\right)$ and $\left(x_{j 2}, 0\right)$ are the coordinates of their corresponding receivers, respectively. We will use the simplex method [20] to find the optimum point. The method requires only function evaluations rather than derivatives as in the conjugate gradient method or steepest decent method. It is not very efficient in terms of the number of function evaluations that it requires. However, it is rather robust compared with the conjugate gradient and/or the steepest decent in that it has some ability to escape from the valley of local minima. Basically, an initial guess of each coordinate of the crack tip is made, then the minimal deviation function for those coordinates is determined and finally the 'true' crack tip coordinates are determined through the optimization process.

At first glance, the maximum and minimum coordinates of all possible crack tips in the first step of the intersecting methods could be used as boundaries using the numerical optimization method, and the resulting estimated crack tips from the second step averaging as initial guesses. Unfortunately, in our numerical experiments, we found that this is true only for the cases where the arrival time errors are rather small, say less than $2 \%$. In the cases where the arrival time errors are higher, the maximum and minimum of all possible crack tips from the first step of the intersecting method are not good enough to even serve as reasonable bounds of the optimum point.

The contour plot of the cost function $F_{5}$ for the cases of the crack tip at $(2,12)$ and $(8,12)$, respectively, with $1 \%$ error in arrival times are shown in Fig. 4. The horizontal axis in the plot corresponds to the surface of 


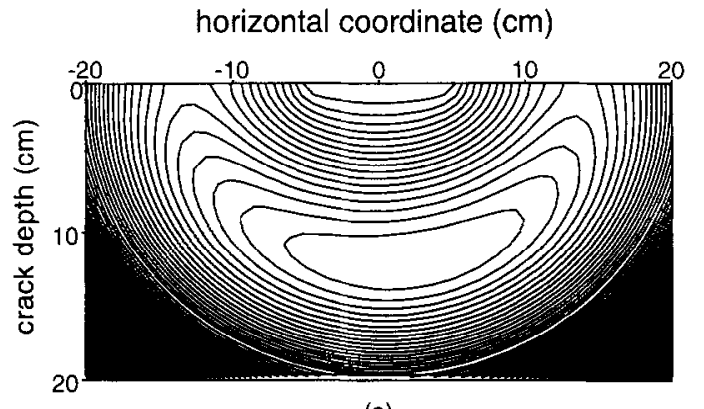

(a)

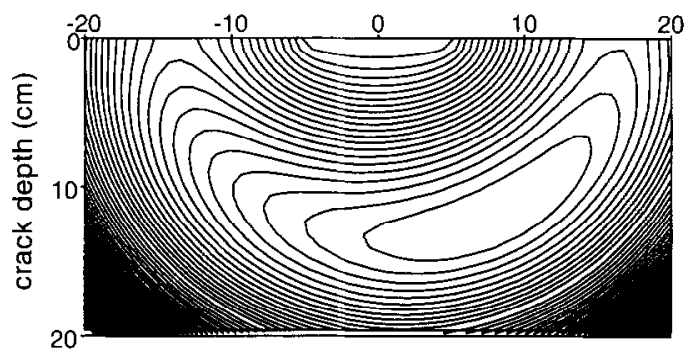

(b)

Fig. 4. Contour plots of error function $F_{5}$, for crack tip at (a) $(2,12)$; (b) $(8,12)$.

the cracked body, while the vertical axis points in towards the body. The contour plots demonstrate that the values of the cost function vary more rapidly in the radial direction than in the angular direction. This suggests that the recovery of the crack length would be more reliable than the crack orientation and/or depth when the error in the arrival time is presented.

\section{Numerical results}

All the cases presented in this section are set up as follows. There are two scts of data per numcrical experiment. In each set of data, a single impact force and four receivers are arranged in such a way that they are on different sides of the crack mouth. In all the numerical examples, the impact forces are locating at $( \pm 5,0) \mathrm{cm}$, respectively, while the receivers are at $( \pm 6,0),( \pm 10,0)$, $( \pm 14,0)$ and $( \pm 18,0) \mathrm{cm}$. The wave speed of the specimen is taken as $4000 \mathrm{~m} \mathrm{~s}^{-1}$. The eight arrival time data (one arrival time per each receiver per one source) are computed according to Eq. (1) and then superimposed by Gaussian errors in order to simulate extracted arrival times from the experimental responses. The relative errors to the exact arrival times are taken as $1 \%, 2 \%$ and $5 \%$, respectively. The crack tip coordinates are then recovered accordingly. Notice that in the current configurations, the exact arrival times are in the order of $70-120 \mu \mathrm{s}$, hence the errors in the arrival times are taken to be in the order of $1-6 \mu \mathrm{s}$.

For all numerical examples performed in this analysis, the results obtained using the intersection of ellipses and intersection of circles, i.e. the methods described in Sections 3.1 and 3.2 are exactly the same. Hence all the numerical results are presented using the intersecting method (either by ellipse or by circle), analytical optimization (of Section 3.3), and numerical optimization (of Section 3.4). The horizontal axis in the plot corresponds to the surface of the cracked body, while the vertical axis is pointed in towards the body. (The actual crack is plotted by the bold line in the figure for reference.)

Figs. 5-7 show the recovered crack tips by three different methods for a slightly inclined crack with a crack tip at $(2,12) \mathrm{cm}$ for various error levels in arrival time data, namely $1 \%$ for Fig. 5, 2\% for Fig. 6, and 5\% for Fig. 7. Since the errors of arrival times were simulated by a random number generator, it is worthwhile to examine the sensitivity of various methods with respect to these random errors on arrival times. We performed the crack tip recovery 100 times for each set up, i.e., 100 random numbers per each level of error. This corresponds to performing 100 experimental tests

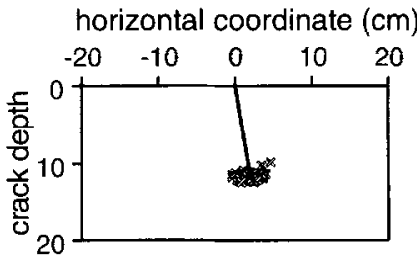

(a)

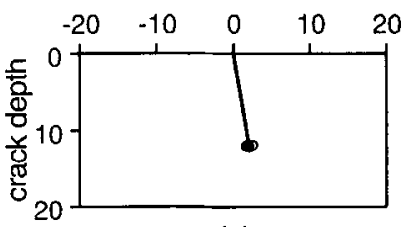

(c) horizontal coordinate $(\mathrm{cm})$

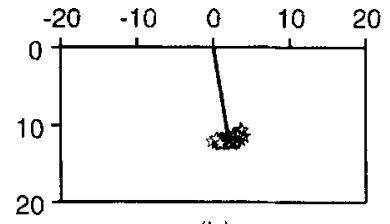

(b)

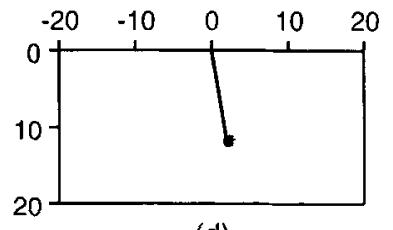

(d)
Fig. 5. Recovered crack tips for crack tip at $(2,12)$ with $1 \%$ error in arrival times by (a) intersection; (b) analytic optimization; (c) numerical optimization; (d) averages of 10 results.

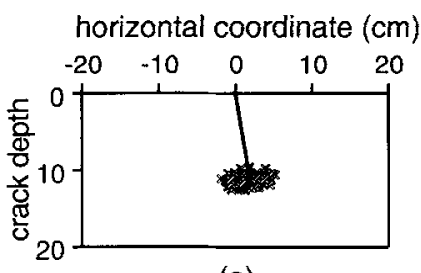

(a)

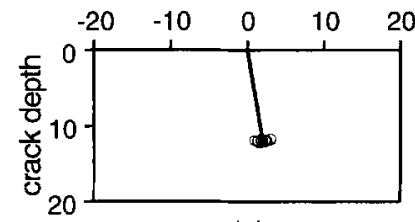

(c) horizontal coordinate (cm)

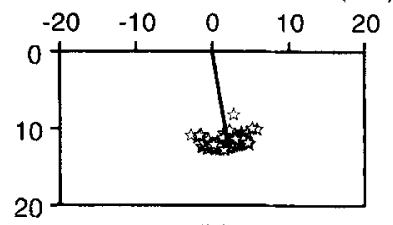

(b)

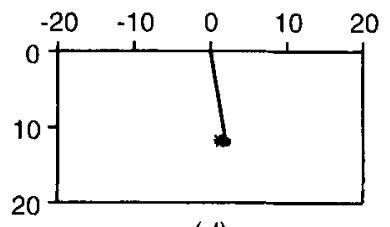

(d)
Fig. 6. Recovered crack tips for crack tip at $(2,12)$ with $2 \%$ error in arrival times by (a) intersection; (b) analytic optimization; (c) numerical optimization; (d) averages of 10 results. 


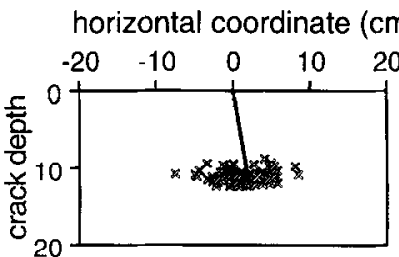

(a)

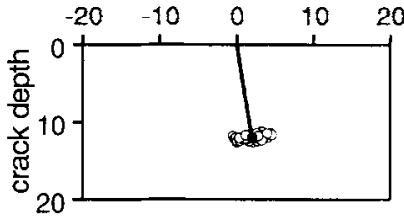

(c) horizontal coordinate $(\mathrm{cm})$

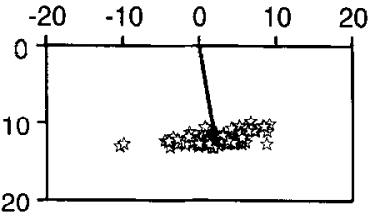

(b)

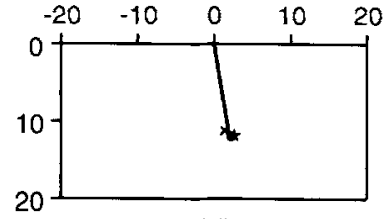

(d)
Fig. 7. Recovered crack tips for crack tip at $(2,12)$ with $5 \%$ error in arrival times by (a) intersection; (b) analytic optimization; (c) numerical optimization; (d) averages of 10 results.

of same level of error per same geometrical and same experimental configuration. The 100 recovered cracks are marked in a light black colour directly at their corresponding position in the subfigures (a)-(c). Subfigure (a) is the result of the intersection method, and (b) and (c) are of the two-step analytic optimization and the numerical optimization methods, respectively. The average of 100 recovered crack tips for each different methods are also marked in dark black colour at their corresponding position in the subfigure for reference. Note that the resulting estimated crack tips from the intersecting method are used as the initial guess in the simplex method, but not as boundaries.

When the error in the arrival time is less than $1 \%$, the performance of the two-step analytic optimization method is about the same as of the intersecting method, if not slightly better. On the other hand, the performance of analytic optimization method deteriorates rather quickly as the error of arrival time becomes larger. Noticeably, the performance of the numerical optimizalion method is superior to the other two methods, regardless the level of errors superimposed on the arrival data. Moreover, the recovered crack length and/or depth for this slightly inclined crack is more robust than the recovered crack orientation for all three methods.

The results of subfigures (a)-(c) also suggest that the average always performs the crack tip recovery very well. Unfortunately, in practice, it is not practical to perform 100 experiments per each specimen. We therefore take 10 experiments per each specimen instead. The averages are marked at their corresponding positions in subfigure (d), where $\times$, $\star$ and correspond to the intersecting method, the two-step analytical optimization method and the numerical optimization method, respectively.

Figs. 8-13 are for the cases where the crack tip is located at $(8,12)$ and $(14,12)$, respectively. Again, the numerical optimization seems more robust than all other

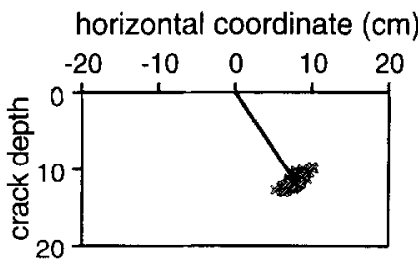

(a)

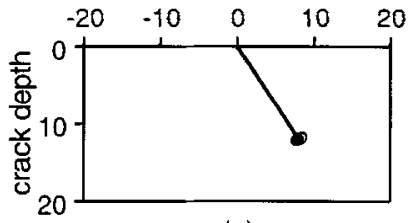

(c) horizontal coordinate $(\mathrm{cm})$

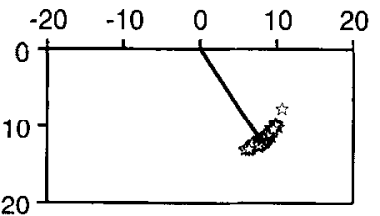

(b)

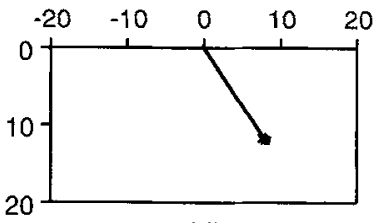

(d)
Fig. 8. Recovered crack tips for crack tip at $(8,12)$ with $1 \%$ error in arrival times by (a) intersection; (b) analytic optimization: (c) numerical optimization; (d) averages of 10 results.

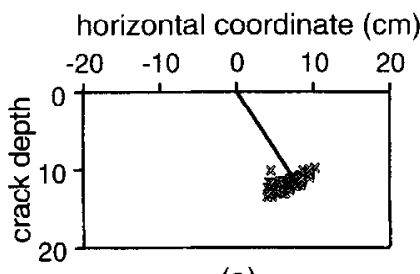

(a)

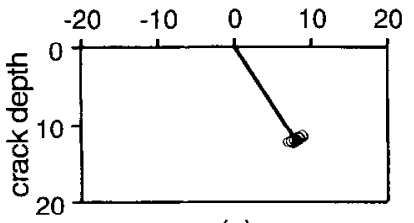

(c) horizontal coordinate $(\mathrm{cm})$

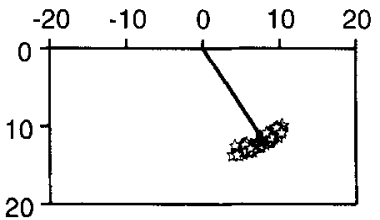

(b)

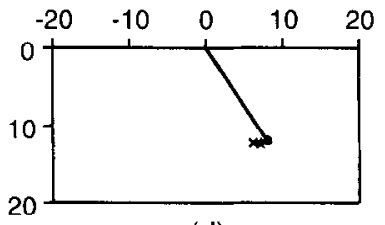

(d)
Fig. 9. Recovered crack tips for crack tip at $(8,12)$ with $2 \%$ crror in arrival times by (a) intersection; (b) analytic optimization; (c) numerical optimization; (d) averages of 10 results.

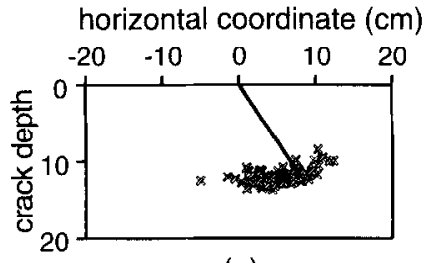

(a)

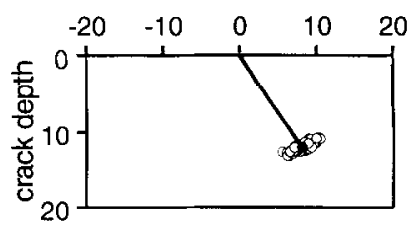

(c) horizontal coordinate $(\mathrm{cm})$

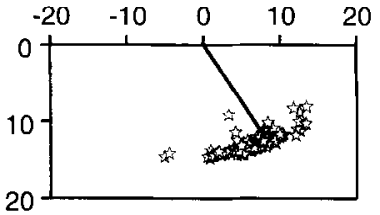

(b)

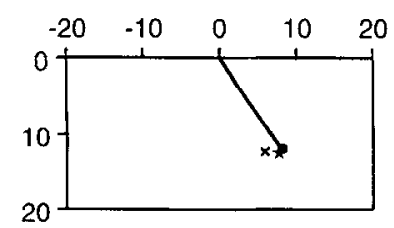

(d)
Fig. 10. Recovered crack tips for crack tip at $(8,12)$ with $5 \%$ error in arrival times by (a) intersection; (b) analytic optimization; (c) numerical optimization; (d) averages of 10 results. 


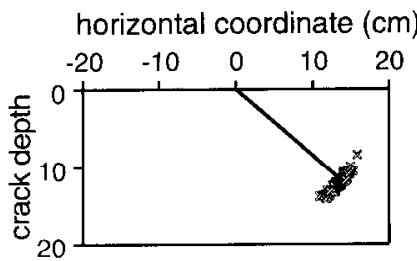

(a)

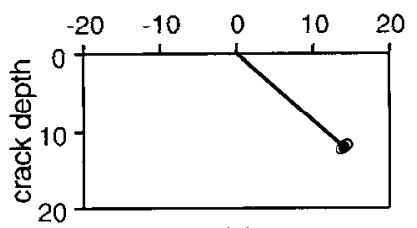

(c) horizontal coordinate $(\mathrm{cm})$

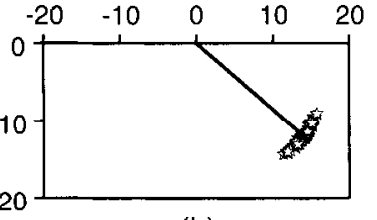

(b)

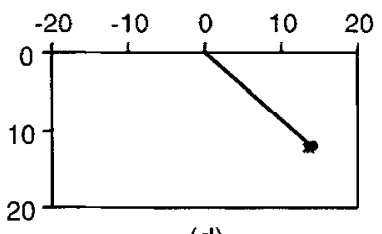

(d)
Fig. 11. Recovered crack tips for crack tip at $(14,12)$ with $1 \%$ error in arrival times by (a) intersection; (b) analytic optimization; (c) numerical optimization; (d) averages of 10 results.

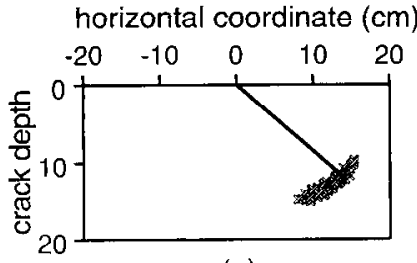

(a)

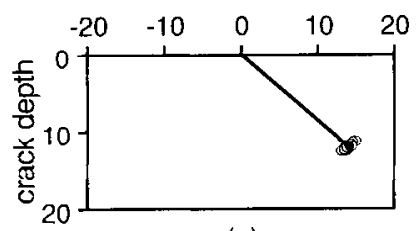

(c) horizontal coordinate $(\mathrm{cm})$

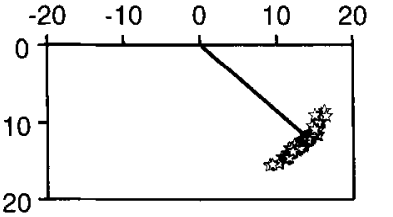

(b)

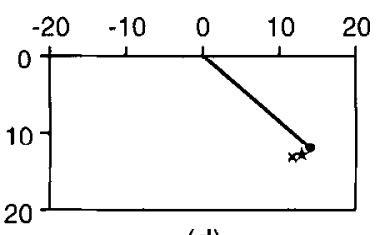

(d)
Fig. 12. Recovered crack tips for crack tip at $(14,12)$ with $2 \%$ error in arrival times by (a) intersection; (b) analytic optimization; (c) numerical optimization: (d) averages of 10 results.

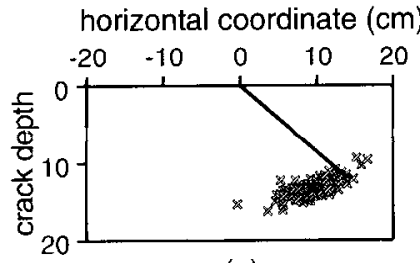

(a)

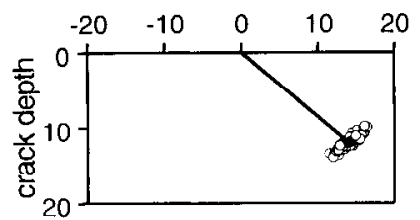

(c) horizontal coordinate $(\mathrm{cm})$

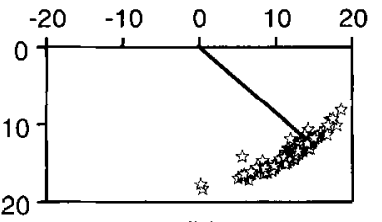

(b)

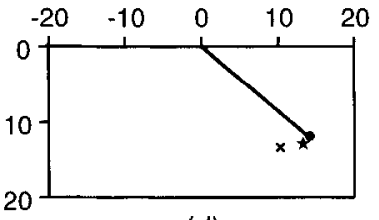

(d)
Fig. 13. Recovered crack tips for crack tip at $(14,12)$ with $5 \%$ error in arrival times by (a) intersection; (b) analytic optimization; (c) numerical optimization; (d) averages of 10 results. methods in all cases. Moreover, the averages of 10 results from the numerical optimization recover the crack tip very successfully. It is of interest to note that the recovered crack length is more robust than the recovered crack depth (and/or orientation), except for the cases of slightly inclined cracks where the crack length and crack depth are not very different from each other. This conclusion is slightly different from the original TOFD method, in which the crack depth rather than length is believed to have been recovered.

\section{Conclusion}

In this paper we have examined four algorithms to recover the crack tips of a surface-breaking line crack from the arrival time information of diffracted waves which are produced by a broad-band transient source such as a dropping steel ball. The exact arrival time from source point through the crack tip then to the receiver is superimposed by Gaussian error in order to simulate the arrival time extracted from the real experimental responses. Three different magnitudes of relative Gaussian error were considered. The performance of different methods were examined by three different geometries of surface-breaking line crack.

The results show that the two intersecting methods yielded the same results, at least in all our numerical studies. The two-step analytical optimization method was the most sensitive to the magnitude of relative error in arrival times, compared to other methods. Numerical optimization always gave the best performance, in robustness as well as accuracy, for all the three methods. In general, the recovered length is more reliable than depth and orientation. This is slightly different from the conclusion of the original TOFD. Finally, an average of several results from numerical optimization method recovered the crack tip very successfully even when the errors on arrival times are up to $5 \%$. This suggests that the proposed algorithms could play an important role, at least as an alternative to TOFD, in crack tip recovery using arrival time data, especially for the cases where impact force is selected as a source and linear scan is not practical.

\section{Acknowledgement}

This work was carried out in the course of research sponsored by the National Science Council of the Republic of China under Grant NSC 84-2621P002-010B. 


\section{References}

[1] J.D. Achenbach, From ultrasonics to failure prevention, in: S.K. Datta, J.D. Achenbach, Y.S. Rajapake (Eds.), Elastic Waves and Ultrasonic Nondestructive Evaluation, Elsevier, New York, 1990 , p. 3.

[2] L. Adler, H.L. Whaley, Interference effect in a multi-frequency ultrasonic pulse echo and its application to flaw characterization, J. Acoust. Soc. Am. 51 (1972) 881.

[3] L. Adler, K.V. Cook, H.L. Whaley, R.W. McClung, Flaw-size measurement in a weld sample by ultrasonic frequency analysis, Materials Evaluation 35 (1977) 44.

[4] M.G. Silk, B.H. Lidington, The potential of scattered or diffracted ultrasound in the determination of crack depths, Non-destructive Testing 8 (1975) 146.

[5] M.G. Silk, B.H. Lidington, Defect sizing using an ultrasonic time delay approach, Br. J. Non-destructive Testing 17 (1975) 33.

[6] B.H. Lidington, M.G. Silk, P. Montgomery, G. Hammond, Ultrasonic measurements of the crack depth of fatigue cracks, Br. J. Non-destructive Testing 18 (1976) 165.

[7] M.G. Silk, B.H. Lidington, An evaluation of single probe bulkwave time-delay techniques in sizing cracks in steel, Non-destructive Testing Int. 10 (1977) 129

[8] M.G. Silk, Interpretation of TOFD data in the light of ASME XI and similar rules, Br. J. Non-destructive Testing 31 (1989) 242.

[9] M.G. Silk, Estimates of the probability of detection of flaws in TOFD data with varying levels of noise, Insight: Non-destructive Testing and Condition Monitoring 38 (1996) 31.

[10] Q. Shan, Surfacc-breaking fatiguc crack detection using laser ultrasound, Appl. Phys. Lett. 62 (1993) 2649.
[11] P.A. Doyle, C.M. Scala, Crack depth measurement by ultrasonics: A review, Ultrasonics 16 (1978) 164.

[12] M. Sansalone, N.K. Carino, Impact-echo method: detecting honeycombing, the depth of surface-opening cracks and ungrouted ducts, Concr. Int.: Design Constr. 10 (1988) 38.

[13] Y. Lin, M. Sansalone, Detecting flaws in concrete beams and columns using the impact-echo method, Mater. J. Am. Concr. Inst. 89 (1992) 394.

[14] Y. Lin, W.C. Su, The use of stress waves for determining the depth of surface-opening cracks in concrete structures, Mater. J. Am. Concr. Inst. 93 (1996) 494.

[15] T.-T. Wu, J.-S. Fan, Crack depth determination of a concrete specimen using phase identification of transient elastic waves, J. Chin. Inst. Civ. Hydraul. Eng. 8 (1996) 337.

[16] P.L. Liu, C.D. Tsai, 'Г.T. Wu, Imaging of surface-brcaking concrete cracks using transient elastic waves, NDT\&E Int. 29 (1996) 323.

[17] A. McNab, I. Dunlop, A review of artificial intelligence applied to ultrasonic defect evaluation, Insight 37 (1995) 11.

[18] T.T. Wu, J.S. Fang, G.Y. Liu, M.K. Kuo, Determination of elastic constants of a concrete specimen using transient elastic waves, J. Acoust. Soc. Am. 98 (1995) 2142.

[19] D.K. Mak, Accuracy in locating a point scatterer in ultrasonic testing - using the TOFD technique. Br. J. Non-destructive Testing 31 (1989) 481 .

[20] W.H. Press, S.A. Teukolsky, W.T. Vetterling and B.P. Flannery, Numerical Recipes in FORTRAN, Cambridge, 1992, p. 402. 